\section{Highlights of the 27th International Papillomavirus Conference and Clinical Workshop: part 3: epidemiology and public health}

Marc Arbyn*, Lutz Gissmann', Achim Schneider² \& Andreas M Kaufmann²

'Deutsches Krebsforschungszentrum, Heidelberg, Germany

${ }^{2}$ Clinic for Gynecology, Charité-Universitätsmedizin Berlin, Campus Benjamin Franklin \& Campus Mitte, Berlin

Germany

*Author for correspondence: Unit of Cancer Epidemiology, Scientific Institute of Public Health.

J Wytsmanstreet 14, B1050 Brussels, Belgium = Tel.: +49 3226425021 = Fax: +49 3226425410

=marc.arbyn@wiv-isp.be

\section{7th International Papillomavirus Conference and Clinical Workshop}

Berlin, Germany, 17-22 September 2011

The 27th International Papillomavirus Conference and Clinical Workshop, held in Berlin (17-22 September 2011) brought together more than 2000 scientists, clinicians and public health experts who shared new findings in the knowledge of the HPV and the prevention and treatment of HPV-related disease. In this last of three reports of the conference, the sessions and workshops dedicated to epidemiology and public health are summarized, which focused on the natural history of HPV infection, risk factors for HPV infection and HPV-associated lesions, descriptions of the current burden and trends of HPV-related cancers, HPV testing in cervical cancer screening and management of women who screen positively, prophylactic HPV vaccination, prevention of cervical cancer in low-resource countries through screening and vaccination, and the prevention and treatment of other cancers caused by HPV. The report ends with the highlights of the 6th Cochrane workshop on systematic reviews regarding prevention of HPV-related disease. The first two parts of the 27th International Papillomavirus conference on Basic Science and Applied Clinical Science were covered in the two previous issues of Future Virology.

\section{Sessions on epidemiology \& public health}

Prophylactic vaccines (A Hildesheim)

A Kreimer presented results from the NCI Costa Rica Vaccine Trial that demonstrated strong efficacy of Cervarix against anal HPV infections among females. C Velicer described a Merck-sponsored post-marketing study to evaluate vaccine safety within a health maintenance cohort in the USA of close to 200,000 vaccinated females, and presented initial results that confirm the overall safety of Gardasil. T Malagon focused on statistical issues and difficulties related to the interpretation of data on measures of vaccine cross-protection. A presentation by J Tota evaluated rates of multiple HPV infections among unvaccinated women and demonstrated that different HPV types do not appear to compete at the infection level, providing some reassurance that HPV vaccination will not result in replacement of vaccine types with nonvaccine types. Evaluation of whether vaccination will result in replacement at the lesional level, however, will require additional follow-up of vaccinated cohorts. B Marshall described a statistical control chart method to monitor long-term vaccine effectiveness in single-arm studies and M Boily described modelling methods to monitor and evaluate the impact of HPV vaccination programs. C Levin reported on costs associated with the introduction of HPV vaccination programs in low-resource settings suggesting that costs can vary between countries, that start-up costs associated with these programs can constitute a significant proportion of expenditures in the initial years, and that the cost of the vaccine itself remains a substantial contribution to the overall cost of vaccine implementation. S Poulsen presented the encouraging results from the general practitioner-based vaccination program in Denmark and demonstrated that high vaccination coverage can be achieved through the use of existing health delivery systems within a country. $S$ Poulsen also reported that high vaccination coverage was achieved through the use of existing general practitioner-based delivery systems in Denmark.

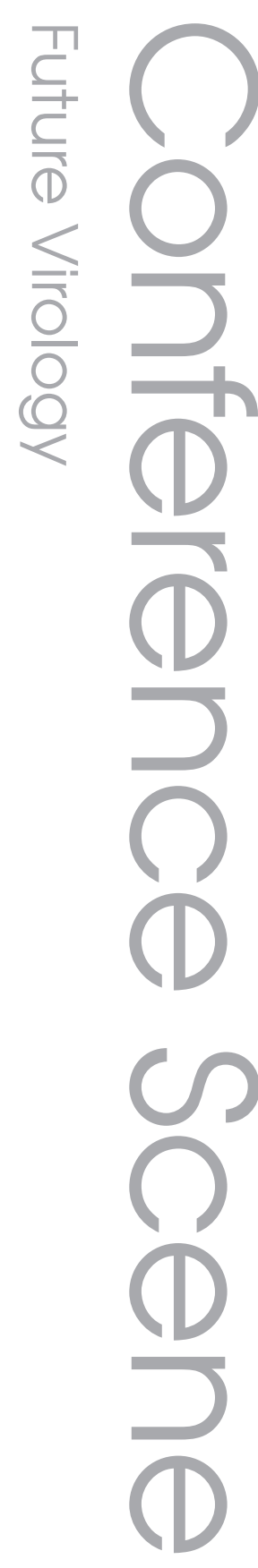

\section{Keywords}

- ano-genital cancer

- cervical cancer $=$ evidencebased medicine $=$ head and neck cancer $=\mathrm{HPV}=$ human papillomavirus $=$ screening " vaccination

\section{Future $\because \%$ Medicine}




\section{Descriptive epidemiology/burden of disease (X Bosch)}

L Bruni and L Alemany presented the correlation of HPV16 in the general population and the corresponding fraction of cervical cancer attributable to HPV16. This project is part of a wider exercise that is attempting to estimate the correlations between HPV and cervical cancer $[1,101]$. G Clifford reviewed the HPV type distribution across the broad spectrum of lesions related to HPV, a useful exercise to inform HPV types to be considered in screening programs. F Carozzi examined the variability of type distribution in a large screening trial in Italy, showing that there is some regional variability but none in relation to age groups. $\mathrm{M}$ Brotons presented a literature review on the burden of genital warts in European countries, reporting a range of incidence rates (per 100,000 women-years) between 100 (Spain) and 3000 (Germany). $\mathrm{T}$ Querec reported some clustering of multiple HPV infections and of HPV types in the general population/referral clinic patients in the USA. L Massad explored the impact of HPV-positive vaginal lesions in patients with HIV, concluding that these infections are common but often represent transient infections. J McCloskey described the screening and diagnostic procedures recommended to evaluate patients referred to an anal cancer clinic due to anal cytological abnormalities. Finally, M Scherpenisse reported on a cross-sectional baseline HPV type-specific serological survey in the Dutch population to be used in the evaluation of the vaccination program in future years.

\section{HPV testing (M Arbyn)}

$\mathrm{K}$ Cuschieri explained the process of setting up a cervical cytology biobank in Scotland, which constitutes an invaluable resource for HPV research and for monitoring the effects of screening and vaccination. The specificity of HPV screening in developing countries could be improved, at the expense of a certain loss in sensitivity, by identifying DNA or the E6 protein of the most important genotypes (HPV16, 18 and 45) (YL Qiao and FH Zhao). Two tests, the COBAS $^{\circledR}-4800$ assay and the Abbott RealTime High-Risk HPV assay, which detect 14 high-risk HPV types and, in addition, also identify HPV16 and 18 , were evaluated. The first fulfils minimal requirements for sensitivity, specificity and reproducibility as defined in European guidelines for cervical cancer screening (B Lloveras) [2], while the second showed similar accuracy to Hybrid Capture-2 (HC2) in a colposcopy setting. Early effects of HPV vaccination can be monitored by HPV genotyping of vaginal or urine samples collected through the Swedish chlamydia trachomatis screening of female adolescents using high-throughput general PCR followed by mass spectrometry (A Söderlund). HPV testing of a self-collected vaginal sample using the Viba-brush ${ }^{\circledR}$ (Rovers Medical Devices BV, The Netherlands) is as accurate as HPV testing of an endocervical sample taken by a clinician (M Dijkstra). An ELISA assay identifying anti-HPV16/18 antibodies was validated for use in the local laboratory involved measuring immunogenicity of the bivalent vaccine in the Costa Rica vaccination trial (A Garcia-Pineres).

\section{Prevention of HPV infection or HPV-related disease (E Franco)}

A Grulich gave an update on the populationlevel impact of vaccination with the quadrivalent vaccine in Australia, showing how it has led to a reduction in the incidence of genital warts among women and indirectly also among men, via herd immunity obtained by decreased transmission. N Schlecht described the preliminary results of cervical and extracervical HPV prevalence among sexually active female teenagers receiving opportunistic HPV vaccination in New York, USA. The expected reduction in prevalence of vaccine-associated types in the cervix was observed as a dose-response trend with number of vaccine doses received. However, the reductions in prevalence for vaccine-target types in noncervical sites (oral and anus) were not significant, raising questions about the benefit of vaccination for mucosal sites other than the cervix. D Watson-Jones described a clusterrandomized trial of school-based HPV vaccine delivery strategies in Tanzania. Both approaches (class-based and age-based) were highly acceptable, but class-based delivery attained higher coverage than the age-based strategy. The study also identified potential predictors of coverage failure; school absenteeism and parental refusal being the most important. Y Flores and M Hernandez-Avila provided an overview of the Mexican government efforts in deploying HPV vaccination and HPV testing in pilot cervical cancer prevention projects across the country. They showed how strong leadership and coordinated action has permitted wide acceptance of both prevention fronts and a coordinated plan for training the many public health stakeholders in vaccination and screening. M Nygard described the baseline prevalence of HPV types in Nordic countries prior to large-scale 
implementation of HPV vaccination. HPV genotyping of liquid-based cytology samples from the screening program was proposed as an ongoing sentinel mechanism to monitor changes in type-specific HPV prevalence. M Madeleine presented the findings from a record-linkage study of transplant recipients in the USA showing how risk of HPV-associated genital lesions is elevated among transplant recipients, but the magnitude of risk varies depending on the organ being transplanted and the type of lesion (in situ versus invasive). D Patel described the treatment patterns for in situ cervical cancer in the Michigan registry, one of the oldest in the USA. Patterns reflected the adoption of evidence-based guidelines for treatment. S Hariri described a surveillance system based on selected sentinel sites in the USA to monitor the impact of HPV vaccination on the rates of type-specific cervical intraepithelial neoplasia (CIN) and other cervical abnormalities.

\section{Cost-effectiveness \& awareness (S Franceschi)}

This session focused on cost-effectiveness and awareness of HPV vaccination programs and cervical cancer screening in high- and low-resource countries. In her introduction, S Franceschi pointed to some recent epidemiological data that indicates the vast benefits of delaying first HPV infection even if vaccine efficacy does not last lifelong.

AC Rodriguez dealt with the impact of HPV16/18 vaccine on screening in the community-based Costa Rica Trial (7466 women, $18-25$ years old). Vaccinated women had fewer high-grade squamous lesions (-12\%) and lowgrade squamous lesions $(-6 \%)$ than unvaccinated women. The positive predictive value of abnormal cytology was also decreased. Colposcopy referral rates slightly diminished among vaccinated women, but the difference increased as years since vaccination increased. $\mathrm{N}$ Van de Velde compared the potential effectiveness of the candidate nonavalent vaccine (HPV16/18/6/11/31/33/45/52/58) with bivalent or quadrivalent vaccines. He suggested that the potential incremental benefits of the nonavalent vaccine would be approximately 10 and $5 \%$ for CIN2+ and squamous-cell cervical carcinoma, respectively. Incremental benefits from the nonavalent vaccine would be twofold larger if the cross-protection of HPV16/18 vaccines against other types was not long-lasting. P Paul reported the concordance of mother-daughter knowledge (536 pairs) on HPV vaccine after a vaccination demonstration project had taken place in Vietnam. Daughters knew more about the HPV vaccine while mothers knew more about cervical cancer. Fully vaccinated girls and their mothers both had better knowledge scores than partially or unvaccinated girls or their mothers. L Eckert focused on the overutilization of HPV testing among young women in the USA. According to an insurance database, HPV testing in 2008 was relatively common in women under 21 years of age (i.e., a group where HPV testing is never recommended). In 21-30-yearold women, the number of HPV tests exceeded the recommended indication by threefold (i.e., women with ASC-US). Nationwide, these findings would translate into approximately US\$51 million overspent per year. W Quentin provided estimates of costs of a school-based HPV vaccination project in Mwanza region, Tanzania. The costs per fully immunized girl were high (about $\$ 52$, vaccines not included) but they should decrease sharply $(\$ 18)$ in a scaledup vaccination program. The presentation by E Conway focused on the impact of male and female vaccination with HPV16/18/6/11 vaccine versus female-only vaccination in Australia. Men having sex with men have high HPV disease burden that will not be decreased by female-only vaccination. The incremental cost-effectiveness of vaccinating males would be substantial for genital warts and, especially, anal cancer (from 15 to $75 \%$ fewer HPV16/18 anal cancers among males). JF Shi and K Canfell described a possible double approach to screening in urban China. It consisted of free-of-charge provision of low-cost $(\leq \$ 10)$ HPV-testing at least once a lifetime to all women. A small subset of women will have access to HC2 and liquid-based cytology at their own expense. Ten-yearly HPV testing showed a very good incremental cost-effectiveness ratio: approximately $\$ 1200$ per life-year saved. Finally, F Carozzi tackled the way biomarkers may improve the cost-effectiveness of primary HPV-based screening in Italy. Assuming that the specificity of cytology (currently the first-choice triage for HPV-positive women) is $60 \%$, a putative biomarker that costs $50 \%$ more than cytology would be cost-saving if its specificity was $80 \%$. If a biomarker costs twice the cytology, its specificity should be $90 \%$.

\section{Noncervical sites (J Smith)}

Evidence has established that HPV plays an etiological role in the development of vaginal, vulvar, penile and oral/oropharyngeal cancers, with most cases being attributable to HPV-type 16. 
Further data are needed for laryngeal, esophageal and lung cancers. However, the exact fraction of the cases of noncervical sites attributable to HPV infection is currently debated, and is largely dependent upon the ability to differentiate noncervical cancer cases that are truly caused by HPV infection from those cases in which HPV infection may simply be a carrier virus. New data showed notable increases in the incidence of oropharyngeal cancer cases in the USA, and estimated that from 2000 to $2004,71 \%$ of cases were attributable to HPV infection (A Chaturvedi). Lower rates of HPV DNA detection were found within oropharyngeal cases $(22 \%)$ in over 2000 formalin-fixed paraffin-embedded head and neck cancers from 26 countries worldwide; additional data will be generated on the prevalence of novel biomarkers (pRB, p16INK4a, p53 and CyclinD1) within this same case series (L Alemany). The medical and economic burden of head and neck cancers was shown to be substantial in Germany, and also to be dependent upon the proportion that is specifically attributable to HPV infections (J Klussman). HPV infection in oral specimens was found in approximately $20 \%$ of MSM in Amsterdam, with higher rates among older, HIV-seropositive men (MF Schim). For esophageal cancer risk, little evidence was found for a causal role of HPV serum antibodies in a large international case-control study (F Sitas). In light of HPV-16 being the primary risk factor for anal cancer, data were presented from the control arm of the HPV prophylactic vaccine trial, which showed that anal HPV infection was common among young women (32\%), and likely acquired by exposure to anal intercourse as well as transmission from the cervix (F Castro). Celecoxib treatment of recurrent respiratory papillomatosis, which is caused by HPV 6 and 11 , is being investigated (A Lucs). Novel serological data indicate that $\beta$-HPV types 8 and 9 may play an etiologic role in the initiation or development of squamous cell skin cancers (J Carter).

\section{Risk factors (M Schiffman)}

HPV-related cancers arise in steps that include viral acquisition, persistence rather than clearance of infection, progression to a precancerous state and invasion. Risk factors alter the rate of a transition step. S Garland described the establishment of a classic twin study designed to address why some women exposed to HPV infection progress to CIN2-3 lesions while others do not. Collaboration was asked to obtain even larger numbers of twins with CIN2-3. $S$ Syrjanen found evidence in a case-control study that smoking might be associated with acquisition and/or persistence of oral HPV infection. A Nyitray studied HPV concordance in monogamous and non-monogamous heterosexual couples and found several interesting associations that differed for type-specific positivity concordance versus concordance on negativity. A Ruiz added to the controversial literature on whether a 'vulnerable period' exists following menarche. They observed that postmenarchal HPV acquisition is particularly likely to lead to CIN2-3/AIS. In a prospective study, A Williamson found that HIV infection leads to increased risk of HPV acquisition and persistence in both women and men. E Roura reported serologic and questionnaire data from the EPIC cohort that supported a role for carcinogenic HPV infections and established risk factors. They discussed whether serologic associations for other STIs provided evidence of causality or residual confounding. O Maranga found that HIV infection elevated the population prevalence of HPV52 and HPV58 relative to HPV16, and is pursuing this finding in women with cervical cancer. H Strickler observed that elevated levels of IGFBP-3 were associated with decreased risk of HPV persistence and CIN2/3, as hypothesized. However, the findings for IGF-1 were not consistent with expectation.

\section{Natural history (X Castellsagué)}

The major focus of this session was the assessment of viral factors in the natural history of HPV infection, CIN and cervical cancer. Data presented all came from well conducted - and mostly large - prospective studies. One study identified local immune response markers being important for HPV clearance versus persistence. Four studies provided precise estimates on HPV incidence, duration, and/or reactivation in young and adult cohorts. A study estimated that about two-thirds of newly detected oncogenic $\mathrm{HPV}$ infections in middle adult-aged women might be attributed to exposure to a recent new partner or multiple partners, and a third to be due to re-detection of a prior infection. Two studies identified HPV persistence and specific genotypes (HPVs 16 and 35) as being strongly related to CIN2+. Finally, data from a large-scale cohort study with 16 years of follow-up showed that HPV negativity predicted long-term low risk of cervical cancer and that persistence of carcinogenic HPV infections increased the risk dramatically. 


\section{Low-resource countries (M Steben)}

A Jaquet presented on how HIV infection was associated with an altered age distribution and increased risk of harboring HR-HPV, which might impact on the subsequent occurrence of cervical cancer and will challenge prevention strategies. S Vaccarella presented the IARC surveys, revealing the heavy burden of HPV infection in certain areas such as Guinea, Nigeria, Mongolia and the Pacific Islands, calling for effective interventions. The 'western' agespecific curve of HPV prevalence should not be taken as the 'natural history of HPV infection'. Vaccination and screening are priorities in countries where HPV prevalence is high, even if no reliable cervical cancer data exist. J Jeronimo discussed screening tools for low-resource settings. careHPVTM (QIAGEN, MD, USA) testing in vaginal self-collected samples shows high sensitivity for CIN3+, with 15\% of women needing further management. If resources are scarce, HPV-E6 or careHPV-16/18/45 testing are alternatives for primary screening or triage of HPV-positive women, but decreased sensitivity is the trade-off. A Lewkowitz showed that the sensitivity of self-sampling for HPV-DNA testing compares favorably with that of liquidbased cytology and visual inspection after application of acetic acid (VIA). Self-sampling for HPV may complement current screening programs by increasing population coverage in low-resource women. S Delany-Moretlwe, P Remes and P Paul showed that adequate information and community education are crucial to increase acceptability of HPV vaccination and to dispel fear and rumors.

\section{Satellite workshops \\ Recent experience with HPV vaccination in Africa, Asia \& Latin America (D Scott LaMontagne)}

This panel included international experts presenting experiences of planning, implementing and evaluating HPV vaccination pilots and programs from low-resource settings. S Wang from WHO gave an overview of the global picture, including a summary of the WHO position paper and guidance documents. D Scott LaMontagne from PATH summarized the experience and results from pilot programs in India, Peru, Uganda and Vietnam, where high coverage was achieved from a variety of acceptable vaccine delivery strategies. L Taper, representing Axios International and the Gardasil Access Program, highlighted the experience from Cameroon, Lesotho and Uganda with small pilots through donations. S Hariri from the US CDC gave a summary of the program being implemented nationally in Rwanda and discussed the challenges in monitoring outcomes. A comprehensive list of planning tools and documents were provided on a USB stick to the participants.

\section{Prioritizing HPV immunization of indigenous populations ( $G$ Wurtak)}

F Cram (Katoa Ltd, New Zealand) described equity tools that have been used in New Zealand to address systemic disparities in health and highlighted the value of inclusive, transparent and accountable processes. E Kliewer (CancerCare Manitoba, Canada) described the higher HPV prevalence and cervical cancer incidence rates, and lower screening and HPV vaccination rates among First Nations peoples in Manitoba, Canada as compared with the general population and suggested the disparity in cervical cancer rates will continue unless vaccination or screening rates improve. P Lhaki (Nepal Fertility Care Center) outlined screening rates, genital wart prevalence and HPV immunization status in Nepal and the challenges currently being experienced regarding immunization policy and practice. B Lawton outlined the cervical cancer disparities between Maori and non-Maori populations in New Zealand and described the roles of the Maori Equity Advisory Group (MEAG), which assisted in achieving and exceeding HPV immunization targets among indigenous peoples. Workshop participants were engaged in small-group discussions regarding methodology to improve HPV vaccination rates in indigenous populations globally.

\section{New approaches to cervical cancer screening in low-resource settings (J Jeronimo)}

This workshop focused in the use of new molecular tests in low-resources settings. J Byamugisha from Makerere University (Uganda) presented results from a multicenter study (START-UP) led by PATH in India, Nicaragua and Uganda showing that careHPV had better sensitivity than Pap smear and VIA. U Rani Poli from MNJ Cancer Institute reported screening using self-collected vaginal samples was very wellaccepted by women and health care providers in those countries; more than $85 \%$ of women agreed to collect a vaginal sample. Finally, J Jeronimo (PATH) reported preliminary results from China, validating a new lateral-flow test 
for E6 protein associated with HPV16/18/45 that achieved a sensitivity of $70 \%$ for CIN3+ with a specificity of $98 \%$ for CIN2+ lesions.

\section{Cervical cancer prevention: best practice from local experts (J Smith)}

Given improvements in prevention tools to prevent cervical cancer worldwide, including the introduction of prophylactic vaccines and HPV diagnostic testing, systematic efforts are needed to address cervical cancer prevention on the local level with evaluation components. Currently, six states in the USA have joined the Cervical Cancer-Free America Initiative, aiming to increase both vaccination and cervical cancer screening rates within their state: Alabama, California, Kentucky, Indiana, North Carolina, and Texas [102]. Local efforts include the development of state-based coalitions to address cervical cancer prevention with a transdisciplinary approach, schoolbased adolescent vaccine demonstration projects, and projects to increase screening among women with a history of infrequent screening. Efforts have also been initiated in Germany to increase cervical cancer knowledge in the general population and among medical specialists, to increase attendance in screening, to educate about primary prevention and to provide consistent, coherent and high quality information on these topics [103]. Lessons learned from these activities will be shared to inform successful strategies for larger programmatic implementation.

\section{Challenges after introduction of HPV vaccines: experiences \& results from HPV post-licensure monitoring in industrial countries (Y Deleré)}

Minimum requirements for monitoring after implementation of HPV vaccination in national vaccination schedules were discussed. After three presentations from countries where HPV vaccination was adopted in the national immunization schedule (USA, England, Germany), the following questions were discussed: What post-implementation activities/programs are absolutely necessary and what is 'scientifically interesting' but without primary public health relevance ('nice-to-have')? For which research questions is international cooperation (e.g., through pooling of data) needed? Is it necessary that each country conducts its own/the same surveillance activities? Summing up, monitoring of vaccine safety and vaccination uptake were considered to be essential to each national program, while it was decided that data on vaccine effectiveness and long-term immunogenicity could be shared or pooled between different countries.

\section{6th Cochrane Workshop: systematic reviews on prevention of HPV-related disease ( $G$ Tortolero-Luna, E Paraskevaidis \& M Arbyn)}

A recent Cochrane review concluded that triage of women with ASC-US cytology using HC2 has higher sensitivity and similar specificity for detection of underlying CIN2+ or CIN3+ compared with repeat cytology, whereas triage of women with LSIL using HC2 is more sensitive but substantially less specific (P MartinHirsch). Meta-analyses show that the risk of preterm delivery in women previously treated for high-grade CIN is higher than in the general population (E Paraskevaidis). However, an increased risk of CIN is not demonstrated when women who received treatment for CIN are compared with women with untreated CIN. A clear correlation of risk of preterm delivery with the depth of excisional treatments suggests large cones $(>12 \mathrm{~mm})$ may be associated with adverse pregnancy outcomes, but small cones are not. The accuracy of HPV-based cervical cancer screening in developing countries with HC2 has been shown to be more sensitive than VIA screening in studies, with improved gold standard verification (M Arbyn). N Wentzensen showed that taking multiple colposcopy-targeted or random biopsies can increase detection of prevalent CIN3 by $30 \%$ compared with one single biopsy, but reproducibility of colposcopic criteria is limited. p16 immunohistochemistry improves the reproducibility and accuracy of cervical histology. J Palewski explained current recommendations for anal cancer screening in high-risk groups $\left(\mathrm{HIV}^{+}\right.$subjects, immunocompromised patients, women with intraepithelial neoplasia) and underlined the need for studies generating evidence.

\section{Conclusion}

This paper highlighted the burden and trends of disease related to HPV infection, causing approximately 600,000 cases of cancer of the cervix, vulva, vagina, anus and oropharynx yearly, as well as other benign disease such as genital warts and recurrent respiratory papillomatosis. Over recent years, the cumulated knowledge of the papillomavirus has generated effective tools for primary prevention of cervical cancer in females and genital warts in females and males. Moreover, screening 
using validated HPV tests has now been shown to be a more effective screening method for cervical cancer precursors than cytology. Numerous other promising applications were discussed, such as the biomarkers potentially distinguishing between progressing and regressing infections and new prophylactic and therapeutic vaccines and chemotherapeutic methods that, in the future, could be used in triage after screening and treatment of HPV-related disease.

The 27th International Papillomavirus Conference and Clinical Workshops was in the tradition of the previous conferences and gathered all communities involved in HPV research. This, along with the past two reports $[3,4]$, summarized the status quo in basic research, clinical application, and policy building by epidemiological and public health studies and underlined the high activity of the field. One important aim was to set the tracks for the new generation of researchers who will soon have to take over responsibility for the future directions of study. This was achieved by offering oral presentation possibilities during the clinical workshops and to give ample oral presentation time for $37 \%$ of all submitted abstracts, especially for the next generation of researchers and young students. In addition to the formal sessions, 14 satellite workshops were organized under the responsibility of individual interest groups and most were already a tradition at this conference. All educational, scientific and satellite workshop sessions were well attended and lively discussions were held. The overall rating 'good to excellent' was $99 \%$ for the clinical workshops and $96.5 \%$ for the main conference, assessed via a web-based survey with a participation of approximately $30 \%$ of total attendees. Importantly, the content of the conference, which was supplied by all the participants as abstract submissions, was rated 'good to excellent' by $96.9 \%$ of all voters, underlining the quality of science presented.

\section{References}

1. Arbyn M, Castellsagué X, de Sanjosé S et al. Worldwide burden of cervical cancer in 2008. Ann. Oncol. 22, 2675-2686 (2011).

2. Meijer CJ, Berkhof J, Castle PE et al. Guidelines for human papillomavirus DNA test requirements for primary cervical cancer screening in women 30 years and older. Int. J. Cancer 124, 516-520 (2009).

3. Arbyn M, Gissmann L, Schneider A, Kaufmann AM. Highlights of the

We invite all interested readers to look forward to the 28th Conference (San Juan, Puerto Rico, 30 November-6 December 2012) [104].

\section{Acknowledgements \\ The authors would like to thank C Schreckenberger for the logistical support in all aspects of the meeting and the correspondence with session chairs as well as the session chairs themselves who made short summaries. Finally, they would like to thank L Rudbert of Destination Oresund and their assistants for having brought together the whole community of HPV experts in the fascinating city of Berlin.}

\section{Financial \& competing interests disclosure}

M Arbyn is supported by (1) the European Commission through (1a) the ECCG project (European Cooperation on development and implementation of Cancer screening and prevention Guidelines, IARC, Lyon, France), funded by DG Sanco, Luxembourg; through (1b) the PREHDICT project (grant No. 242061, coordinated by the Vrije Universiteit Amsterdam, The Netherlands) and (1c) through HPV-AHEAD (coordinated by $I A R C)$, funded by the 7th Framework Programme of DG Research, Brussels; (2) the Belgian Foundation Against Cancer (Brussels, Belgium). L Gissmann is a consultant to Qiagen, GlaxoSmithKline (GSK) and Sanofi Pasteur MSD and, due to existing intellectual property, receives royalties from sales of Gardasi ${ }^{\circledR}$ and Cervarix ${ }^{\circledR}$. A Schneider received honoraria from GSK, Sanofi Pasteur and Karl Storz for advisory and speaking activities. AM Kaufmann has received travel grants and speakers honoraria from GSK and SP-MSD. In the past 5 years he has served as advisory board member for GSK and Gen-Probe. The authors have no other relevant affiliations or financial involvement with any organization or entity with a financial interest in or financial conflict with the subject matter or materials discussed in the manuscript apart from those disclosed.

No writing assistance was utilized in the production of this manuscript.

27th International Papillomavirus Conference and Clinical Workshop: part 1. Future Virol. 6, 1389-1396 (2011).

4. Arbyn M, Schneider A, Gissmann L, Kaufmann AM. Highlights of the 27th International Papillomavirus Conference and Clinical Workshop: part 2: applied clinical science. Future Virol. 7, 19-24 (2012).

\section{Websites}

101. WHO/ICO (Institut Català d'Oncologia) Information Centre on HPV and Cervical
Cancer (2011).

www.who.int/hpvcentre

102. Cervical Cancer Free America. www.cervicalcancerfreeamerica.org

103. Information and education about cervical cancer and HPV. www.zervita.org

104. 28th International Papillomavirus Conference and Clinical Workshop, San Juan, Puerto Rico, 30 November-6 December 2012. www.hpv2012pr.org 